\title{
The state of minimally invasive pancreaticoduodenectomy in Chinese mainland: A systematic literature review
}

\author{
Jianyi Ding ${ }^{1,2}$, Chengwu Zhang ${ }^{2}$, Dongsheng Huang ${ }^{2}$, Yuhua Zhang ${ }^{2, *}$ \\ 'Zhejiang Chinese Medical University, Hangzhou, Zhejiang, China; \\ ${ }^{2}$ Division of Hepatobiliary and Pancreatic Surgery and Minimally Invasive Surgery, Zhejiang Provincial People's Hospital, \\ People's Hospital of Hangzhou Medical College, Hangzhou, Zhejiang, China.
}

\begin{abstract}
Summary The development of Minimally invasive pancreaticoduodenectomy (MIPD) in Chinese mainland has been extremely quick. However, the safety and oncologic outcomes remain controversial. This review evaluates the current status of MIPD in Chinese mainland. A systematic literature search was performed using: Pubmed, Web of Sci, CNKI, Wanfang Data and Sinomed databases to filter all studies published up to and including June 2019 using key words "pancreaticoduodenectomy," or "Whipple operation" combined with "laparoscopy," or "laparoscopic," or "robotic," or "da Vinci," or "minimally invasive," or "hand-assisted". This systematic review included 39 articles that documented 2,653 MIPDs in Chinese mainland. The weighted average operative time was $370.6 \mathrm{~min}$, and the weighted average blood loss was $278.0 \mathrm{~mL}$. The overall morbidity was $31.9 \%$, which Clavien-Dindo $\geq$ 3 complications accounted for $13.4 \%$. Pancreatic fistula, delayed gastric emptying, bile leak and postoperative hemorrhage were reported in $20.9 \%, 5.5 \%, 3.5 \%$ and $6.0 \%$ of patients respectively. The average lengh of hospital stay was 16.1 days. The overall surgical mortality was $\mathbf{1 . 7 \%}$. The mean number of harvested lymph nodes was 13.5 , and the rate of positive margin was $5.3 \%$. Based on Chinese national condition, the operative volume of MIPD in Chinese mainland is the leading position in the world, and compared with some large international meta-analysis, no inferior perioperative and short-term oncological outcomes were observed in MIPD of Chinese mainland. However, research on survival analysis and phased learning curve outcomes is needed urgently before the innovative techniques are widely accepted.
\end{abstract}

Keywords: Minimally invasive surgery, pancreaticoduodenectomy, Chinese mainland, laparoscopy, robot

\section{Introduction}

Pancreaticoduodenectomy (PD), as the only potentially curative option in patients with periampullary malignancy, has been recognized as one of the most complicated and risky procedures in general surgery for the past 100 years (1). With the development of

Released online in J-STAGE as advance publication December 24, 2019.

*Address correspondence to:

Dr. Yuhua Zhang, Division of Hepatobiliary and Pancreatic Surgery and Minimally Invasive Surgery, Zhejiang Provincial People's Hospital, People's Hospital of Hangzhou Medical College, No. 158 of Shangtang Road, Hangzhou 310014, China.

E-mail: zhangyuhua1013@126.com surgery technology, the first case of minimally invasive pancreaticoduodenectomy (MIPD) was described in 1994 by Gagner (2). However, its challenging anatomical and anastomotic techniques and inferior short-term outcomes slowed the acceptance of this operation.

After the first report of MIPD in Chinese mainland in 2003 (3), more challenge-pursing and innovative Chinese surgeons have contributed to explore better approaches and procedures with the introduction of advanced technologies.

Previous literature listed advantages of minimally invasive surgery in other fields of general surgery including lower blood loss, faster post operation recovery and comparable oncology outcomes (4). Unlike previous years, more and more small-sample studies have been documented in recent years, which marks attempts from 
non-specialized centers.

In this paper, we reviewed the literature describing MIPD in Chinese mainland to provide a comprehensive evaluation of the current status, focusing on technical details and short-term outcomes of minimally invasive approaches.

\section{Materials and Methods}

\subsection{Definitions and surgical techniques}

MIPD included two main methods: laparoscopic pancreaticoduodenectomy (LPD) and robotic pancreaticoduodenectomy (RPD). Moreover, it could be further classified based on techniques used in resection and reconstruction:

1) Pure LPD, where the entire operation is completed with the assistance of laparoscopic technique;

2) Hand-assisted LPD, where a mini hand port is added to facilitate the procedure;

3) Laparoscopy-assisted LPD, where resection is carried out laparoscopically and reconstruction is completed using a small mini-laparotomy incision;

4) Robotic-assisted PD, where the entire operation or some parts of dissection and reconstruction are performed with the assistance of the da Vinci surgical system.

\subsection{Literature review}

A systematic literature search was performed using: Pubmed, Web of Sci, CNKI, Wanfang Data and Sinomed databases to filter all studies published up to and including June 2019 using key words "pancreaticoduodenectomy," or "Whipple operation" combined with "laparoscopy," or "laparoscopic," or "robotic," or "da Vinci," or "minimally invasive," or "hand-assisted." Relevant articles identified by cross-referencing were also retrieved and reviewed.

\subsection{Inclusion criteria}

Articles describing MIPD in Chinese mainland containing more than 10 cases were included. If patient data was documented more than once from the same institution, the most informative or recent article was considered to prevent data overlap.

\subsection{Exclusion criteria}

Articles lacking original data or missing lots of outcomes, studies referring to animals and cadavers, technique articles, multimedia literature, Chinese articles without English abstracts and academic degree articles were excluded.

\subsection{Data extraction}

All the retrieved studies that met the inclusion and exclusion criteria were independently reviewed by two authors (Ding JY and Zhang YH). Discrepancies between the two reviewers were resolved by discussion.

The variables extracted from the included studies were as follows:

1) Basic information (first author, publication year, study period, number of cases);

2) Technical details(surgical procedures, management of pancreatic stump, management of gastroduodenal artery, specimen extraction site, anastomotic technique in gastroenterostomy, suture technique in choledochojejunostomy, vascular resection and reconstruction);

3) Intraoperative outcomes (operative time, intraoperative blood loss, conversion rate, transfusion rate);

4) Short-term outcomes (overall morbidity, pancreatic fistula, usage of International Surgical Group of Pancreatic Fistula or not, delayed gastric emptying, bile leak, postoperative hemorrhage, length of postoperative hospital stay, Clavien-Dindo $\geq 3$ complications, reoperation, surgical mortality);

5) Oncologic outcomes (malignancy rate, usage of tumor-node-metastasis stage or not, number of harvested lymph nodes, rate of margin negative resection).

\subsection{Statistical analysis}

A weighted average (WA) was used to express the statistical weighted mean of different variables:

$$
\mathrm{WA}=\left(w_{1} x_{1}+w_{2} x_{2}+\ldots+w_{\mathrm{n}} x_{\mathrm{n}}\right) /\left(w_{1}+w_{2}+\ldots+w_{\mathrm{n}}\right)
$$

where $w$ is the number of cases in a publication and $x$ is the mean of a specific variable. The $x$ and its corresponding $w$ are excluded if the variables in some studies are absent or not able to calculate.

The chi-square test was used to compare categorical variables between groups. The Student's unpaired $t$ test or Mann-Whitney $U$ test was used to compare continuous variables, as appropriate. Although this statistical method is not entirely rigorous, the results could be presented through a more intuitive way and some authors including Gumbs (5) and Boggi (6) have already applied this statistical method. Statistical analyses were finished by SPSS statistical software package (version 25.0, SPSS Inc., Chicago, IL, USA). A $p$ value $<0.05$ was considered statistically significant.

\section{Results}

This systematic review included 39 articles that documented a total of 2,653 MIPDs (Table 1) (7-45). Seventeen articles were published between 2010 and 2017, documenting 540 cases (20.4\%), whereas twentytwo were published in the next two years, reporting on $2,113$ cases $(79.6 \%)$ (Figure 1$)$. 
Table 1. Summary of current articles on minimally invasive pancreaticoduodenectomy in more than 10 cases

\begin{tabular}{|c|c|c|c|c|}
\hline First author (Ref.) & Publication year & Study period & Methods & Cases \\
\hline Chao Lu (32) & 2018 & 2012-2017 & PL & 320 \\
\hline Xueqing Liu (30) & 2018 & 2013-2017 & PL & 300 \\
\hline Yunqiang Cai (9) & 2018 & $2015-2018$ & PL & 238 \\
\hline Hang Zhang (43) & 2018 & 2014-2016 & PL & 202 \\
\hline Wei Chai (10) & 2019 & 2015-2017 & $\mathrm{PL} / \mathrm{LA}$ & $102 / 86$ \\
\hline Qingchun Li (27) & 2019 & 2014-2017 & PL & 134 \\
\hline Xiaohui Duan (17) & 2017 & 2014-2017 & PL & 101 \\
\hline Guolin Li (25) & 2018 & 2016-2017 & PL & 100 \\
\hline Tao Zhang (45) & 2018 & 2012-2016 & RA & 100 \\
\hline Yong An (7) & 2019 & 2017-2018 & PL & 90 \\
\hline Yun Liang (28) & 2019 & 2015-2018 & PL & 82 \\
\hline Defei Hong (19) & 2016 & 2013-2016 & PL,RA & 80 \\
\hline Yusheng Du (16) & 2019 & 2016-2017 & PL & 67 \\
\hline Shi Chen (14) & 2015 & 2010-2013 & RA & 60 \\
\hline Fangkuan Li (24) & 2017 & $2012-2016$ & PL & 50 \\
\hline Peng Chen (13) & 2018 & $2015-2018$ & PL, RA & 40 \\
\hline Ronggui Lin (29) & 2018 & $2017-2017$ & PL, RA, HA & 35 \\
\hline Jianjun Li (26) & 2013 & 2002-2012 & PL, LA & 34 \\
\hline Menghua Dai (15) & 2018 & 2016-2018 & PL & 34 \\
\hline Zhigang Wei (37) & 2018 & 2015-2018 & PL & 33 \\
\hline Fan Yang $(J L)(41)$ & 2018 & $2017-2017$ & PL & 30 \\
\hline Rong Tang (34) & 2017 & $2010-2013$ & LA & 29 \\
\hline Fan Yang (CQ) (42) & 2019 & $2016-2018$ & PL & 29 \\
\hline Jianhui Chen (12) & 2016 & 2012-2014 & LA & 25 \\
\hline Zuguang Wu (39) & 2017 & 2014-2016 & PL & 22 \\
\hline Lei Zhang (44) & 2019 & $2015-2018$ & PL & 21 \\
\hline Jiacheng Wu (38) & 2018 & 2017-2017 & PL & 21 \\
\hline Zhao Liu (31) & 2015 & 2011-2012 & LA & 21 \\
\hline Qiang Huang (22) & 2017 & 2016-2016 & PL & 20 \\
\hline Qiuya Wei (36) & 2016 & 2013-2015 & PL & 19 \\
\hline Wentao Gao (18) & 2017 & 2016-2016 & PL & 18 \\
\hline Hai Hu (20) & 2019 & $2015-2018$ & RA & 18 \\
\hline Qinzheng Bai (8) & 2016 & 2015-2016 & LA & 16 \\
\hline Jinmeng Hu (21) & 2018 & 2015-2017 & PL & 16 \\
\hline Huanwei Chen (11) & 2019 & 2015-2018 & PL/LA & $10 / 5$ \\
\hline Mingsheng Sun (33) & 2015 & 2010-2015 & LA & 12 \\
\hline Jun Xu (40) & 2010 & 2005-2008 & LA, HA & 12 \\
\hline Hongbo Wei (35) & 2014 & $2012-2013$ & PL & 11 \\
\hline Wu Ji (23) & 2014 & $2010-2012$ & RA & 10 \\
\hline
\end{tabular}

PL, pure laparoscopic pancreaticoduodenectomy. LA, laparoscopy-assisted pancreaticoduodenectomy. RA, robotic-assisted pancreaticoduodenectomy. HA, hand-assisted pancreaticoduodenectomy. JL, Jilin Province. CQ, Chongqing City.

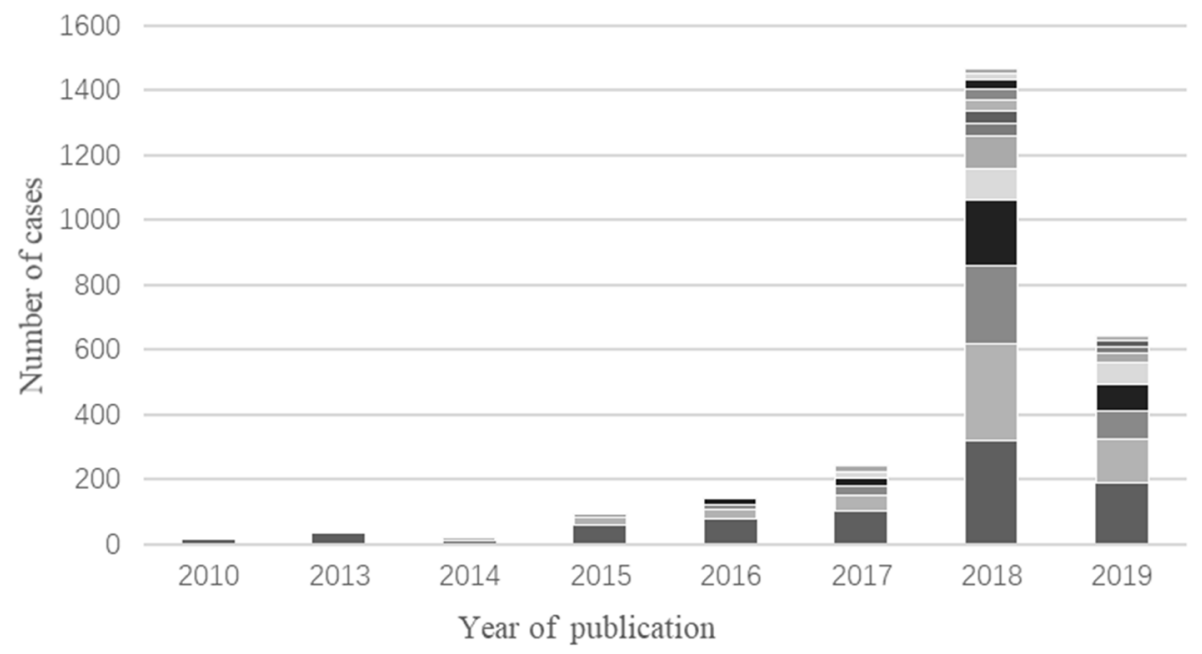

Figure 1. Number of cases documented yearly. The different depth of gray color in each bar represent different studies in that year and their heights mean the sample size of the corresponding study. 
Table 2. Management of pancreatic stump in minimally invasive pancreaticoduodenectomy

\begin{tabular}{|c|c|c|c|c|}
\hline \multirow{2}{*}{ First author (Ref.) } & \multicolumn{4}{|c|}{ Management of pancreatic stump } \\
\hline & PJ & PG & Technique & Stent \\
\hline Chao Lu (32) & 320 & 0 & [E-to-S and D-to-M] & Selectively \\
\hline Xueqing Liu (30) & $295^{\mathrm{a}}$ & 0 & 30 [E-to-E and Invaginating], 265[E-to-S and D-to-M] & Selectively \\
\hline Yunqiang Cai (9) & 238 & 0 & [E-to-S and D-to-M] & Yes \\
\hline Hang Zhang (43) & 202 & 0 & [E-to-S and D-to-M] & Yes \\
\hline Wei Chai $(10)$ & 102(PL), 86(LA) & 0 & $102[$ E-to-S and D-to-M], 86[E-to-S and Invaginating] & Yes \\
\hline Qingchun Li (27) & 134 & 0 & [E-to-S and D-to-M] & Yes \\
\hline Xiaohui Duan (17) & 101 & 0 & [E-to-S and D-to-M] & Yes \\
\hline Guolin Li (25) & 100 & 0 & [E-to-S and D-to-M] & Selectively \\
\hline Tao Zhang (45) & 100 & 0 & [E-to-S and D-to-M] & Yes \\
\hline Yong An (7) & 90 & 0 & [E-to-S and D-to-M] & NA \\
\hline Yun Liang (28) & 82 & 0 & [E-to-S and D-to-M] & NA \\
\hline Dfei Hong ${ }^{\mathrm{b}}$ (19) & 70 & 7 & $70[$ E-to-S and D-to-M], 7[E-to-S and Invaginating $]$ & Yes \\
\hline Yusheng Du (16) & 67 & 0 & $67[$ E-to-S and D-to-M] & Yes \\
\hline Shi Chen (14) & 59 & $1^{\mathrm{c}}$ & $59[$ E-to-S and D-to-M $]$ & Yes \\
\hline Fangkuan Li (24) & 50 & 0 & [E-to-S and D-to-M] & NA \\
\hline Peng Chen (13) & 40 & 0 & [E-to-S and D-to-M] & Yes \\
\hline Ronggui Lin (29) & 35 & 0 & NA & NA \\
\hline Jianjun Li (26) & 34 & 0 & [E-to-S and D-to-M] & Yes \\
\hline Menghua Dai (15) & NA & NA & NA & NA \\
\hline Zhigang Wei (37) & NA & NA & NA & NA \\
\hline Fan Yang (JL) (41) & 30 & 0 & [E-to-S and D-to-M] & Yes \\
\hline Rong Tang (34) & NA & NA & NA & NA \\
\hline Fan Yang (CQ) (42) & 29 & 0 & [E-to-S and D-to-M] & Yes \\
\hline Jianhui Chen (12) & NA & NA & NA & NA \\
\hline Zuguang Wu (39) & 22 & 0 & [E-to-S and D-to-M] & NA \\
\hline Lei Zhang (44) & 21 & 0 & [E-to-S and M-to-M] & Selectively \\
\hline Jiacheng Wu (38) & 21 & 0 & [E-to-S and D-to-M] & Yes \\
\hline Zhao Liu (31) & 21 & 0 & [E-to-S and D-to-M] & Yes \\
\hline Qiang Huang (22) & 20 & 0 & [E-to-S and D-to-M] & Yes \\
\hline Qiuya Wei (36) & 19 & 0 & [E-to-S and D-to-M] & Yes \\
\hline Wentao Gao (18) & 18 & 0 & [E-to-S and D-to-M] & NA \\
\hline Hai Hu (20) & 18 & 0 & NA & NA \\
\hline Qinzheng Bai (8) & 16 & 0 & [E-to-S and D-to-M] & Yes \\
\hline Jinmeng $\mathrm{Hu}(21)$ & 16 & 0 & [E-to-S and D-to-M] & Yes \\
\hline Huanwei Chen (11) & $5(\mathrm{PL}), 10(\mathrm{LA})$ & 0 & [E-to-S and D-to-M], [E-to-S and D-to-M] & NA, Yes \\
\hline Mingsheng Sun (33) & NA & NA & NA & NA \\
\hline Jun Xu (40) & 12 & 0 & [E-to-E and Invaginating] & Selectively \\
\hline Hongbo Wei (35) & 11 & 0 & [E-to-E and Invaginating] or [E-to-S and D-to-M] & NA \\
\hline Wu Ji (23) & 10 & 0 & [E-to-S and D-to-M] & Yes \\
\hline
\end{tabular}

${ }^{a}$ Another 5 cases underwent total pancreatectomy. ${ }^{b}$ Another 3 cases were not described due to conversion to laparotomy. ${ }^{c}$ Pancreaticogastrostomy was not described in detail. PL, pure laparoscopic pancreaticoduodenectomy. LA, laparoscopy-assisted pancreaticoduodenectomy. PJ, pancreatojejunostomy. PG, pancreatogastrostomy. NA, not applicable. D-to-M, duct-to-mucosa. E-to-E, end-to-end. E-to-S, end-to-side. JL, Jilin Province. CQ, Chongqing City.

Pure laparoscopic pancreaticoduodenectomy (PL) have held a dominant position in MIPD according to the literature of recent years. 2070 PL (78.0\%) were documented in our review. And 7 authors $(8,10$ $12,31,33,34)$ describing 194 cases $(7.3 \%)$ laparoscopyassisted pancreaticoduodenectomy (LA).

Robotic-assisted pancreaticoduodenectomy (RA) was gradually put into application for pancreatic surgery in the recent few years. 188 cases (7.1\%) RA were included in our analysis, while only 5 cases $(0.2 \%)$ hand-assisted pancreaticoduodenectomy (HA) of two articles $(29,40)$ are mentioned in our review.

In addition, seven authors applied more than one technique $(10,11,13,19,26,29,40)$, and three of them $(13,19,29)$ simultaneous applied LPD and RPD without data separation. Therefore, the three articles were excluded either in comparison of LPD and RPD or in classification of technique.

\subsection{Technical details}

Technical details of MIPD are listed in Table 2 and Table 3, including management of pancreatic stump, closure of gastroduodenal artery, specimen extraction site, anastomotic technique in gastroenterostomy, suture technique in choledochojejunostomy and vascular resection and reconstruction.

Details on management of pancreatic stump were provided in 34 articles $(87.2 \%)$. Pancreatojejunostomy (PJ) was the major selection to manage pancreatic stump while pancreatogastrostomy(PG) was only mentioned in 8 cases of 2 articles $(14,19)$ and no duct occlusion was reported. Details about anastomosis methods were described in 32 articles (82.1\%), including end-to-end 
Table 3. Technical details of minimally invasive pancreaticoduodenectomy

\begin{tabular}{|c|c|c|c|c|c|}
\hline First author (Ref.) & Management of GDA & Extraction site & $\begin{array}{l}\text { Anastomotic technique } \\
\text { in gastroenterostomy }\end{array}$ & $\begin{array}{c}\text { Suture technique in } \\
\text { choledochojejunostomy }\end{array}$ & $\begin{array}{l}\text { Vascular resection } \\
\text { and reconstruction }\end{array}$ \\
\hline Chao Lu (32) & Clips & NA & Stapled & Selectively & NA \\
\hline Xueqing Liu (30) & Clips or Ligature & Subxiphoid & $\begin{array}{c}\text { Stapled }(68.7 \%), \\
\text { Hand-sewn }(31.3 \%)\end{array}$ & $\mathrm{RS}$ & 10 \\
\hline Yunqiang Cai (9) & NA & NA & NA & NA & 0 \\
\hline Hang Zhang (43) & Clips & Infra-umbilical & Stapled & Selectively & 2 \\
\hline Wei Chai (10) & NA & Subxiphoid & NA & NA & NA \\
\hline Qingchun Li (27) & NA & Umbilical & NA & NA & NA \\
\hline Xiaohui Duan (17) & Ligature and Clips & Infra-umbilical & Stapled & Selectively & NA \\
\hline Guolin Li (25) & Ligature & NA & Stapled & $\mathrm{RS}$ & NA \\
\hline Tao Zhang (45) & NA & Umbilical & NA & NA & 0 \\
\hline Yong An (7) & NA & NA & Stapled & $\mathrm{RS}$ & 3 \\
\hline Yun Liang (28) & NA & NA & NA & NA & NA \\
\hline Defei Hong (19) & NA & NA & NA & NA & 5 \\
\hline Yusheng Du (16) & NA & NA & NA & RS & NA \\
\hline Shi Chen (14) & Ligature & NA & NA & Selectively & 3 \\
\hline Fangkuan Li (24) & Clips & NA & Stapled & RS & NA \\
\hline Peng Chen (13) & Ligature & NA & Stapled & NA & NA \\
\hline Ronggui Lin (29) & Clips & NA & NA & NA & NA \\
\hline Jianjun Li (26) & Ligature & Subxiphoid & NA & NA & NA \\
\hline Menghua Dai (15) & NA & NA & NA & NA & 2 \\
\hline Zhigang Wei (37) & Clips & NA & NA & NA & NA \\
\hline Fan Yang (JL) (41) & NA & NA & Hand-sewn & NA & 1 \\
\hline Rong Tang (34) & NA & Subxiphoid & NA & NA & NA \\
\hline Fan Yang (CQ) (42) & Clips & NA & NA & $\mathrm{RS}$ & NA \\
\hline Jianhui Chen (12) & NA & Subxiphoid & NA & NA & 0 \\
\hline Zuguang Wu (39) & NA & Infra-umbilical & Stapled & NA & 0 \\
\hline Lei Zhang (44) & NA & Subxiphoid & NA & NA & NA \\
\hline Jiacheng Wu (38) & NA & Infra-umbilical & Stapled & $\mathrm{RS}$ & 0 \\
\hline Zhao Liu (31) & Clips & Subxiphoid & NA & IS & 0 \\
\hline Qiang Huang (22) & NA & NA & NA & NA & 0 \\
\hline Qiuya Wei (36) & NA & NA & Stapled & RS & 0 \\
\hline Wentao Gao (18) & NA & NA & Hand-sewn & $\mathrm{RS}$ & 0 \\
\hline Hai Hu (20) & Ligature & Subxiphoid & NA & NA & 0 \\
\hline Qinzheng Bai (8) & Clips & Subxiphoid & NA & NA & NA \\
\hline Jinmeng $\mathrm{Hu}(21)$ & NA & NA & NA & NA & 0 \\
\hline Huanwei Chen (11) & Ligature and Clips & $\begin{array}{l}\text { Subxiphoidv (LA), } \\
\text { Suprapubicv (PL) }\end{array}$ & Stapled & $\mathrm{RS}$ & 0 \\
\hline Mingsheng Sun (33) & Clips & Subxiphoid & NA & NA & 0 \\
\hline Jun Xu (40) & NA & Subxiphoid & NA & NA & 0 \\
\hline Hongbo Wei (35) & NA & NA & NA & NA & NA \\
\hline Wu Ji (23) & NAhi & Subxiphoid & NA & NA & NA \\
\hline
\end{tabular}

GDA, gastroduodenal artery. RS, running suture. IS, interrupted suture. NA, not applicable. JL, Jilin Province. CQ, Chongqing City.

and invaginating $\mathrm{PJ}$, end-to-side and invaginating $\mathrm{PJ}$, end-to-side and duct-to-mucosa PJ, end-to-side and invaginating PG according to Barreto's classification (46). Among the above methods, the end-to-side and ductto-mucosa PJ was the most popular one. Undoubtedly, duct-to-mucosa anastomosis is the most difficult point in PJ. Hence, some modified or innovative methods such as purse-string, Bing's anastomosis (9) or Hong's singlestitch method (47) were applied for further reinforcing the junction between pancreatic duct and intestinal wall $(9,10,16,19,21,41)$. Nevertheless, various modified methods haven't been marked in the tables so as to simplify our classification. As for stent for pancreatic duct, 25 authors (64.1\%) implied entirely or selectively in their studies.

Seventeen authors (43.6\%) described the closure of gastroduodenal artery (GDA). The methods contain clips
(9 articles) or ligature (5 articles). In addition, Duan et al. (17) and Chen et al. (11) applied clips plus ligature while Liu et al. (30) used clips or ligature. Specimen extraction site was described in 19 articles (48.7\%). Chen et al. (11) applied two different incisions to extract specimens in different surgical procedures. 13 authors $(68.4 \%)$ used a subxiphoid incision to extract specimens. In 4 articles $(21.1 \%)$, the specimen was delivered through an infraumbilical incision or enlarged port, in 2 articles $(10.5 \%)$ through an umbilical incision or enlarged port. Besides, suprapubic incision was used by Chen et al. as the second method. Seven authors described the jejunal loop used for duodenal or gastric anastomosis (17.9\%), and all patients in their studies followed an antecolic route. The retromesenteric and retrocolic routes were hardly employed according to the data we pooled. Anastomotic technique in gastroenterostomy and suture technique 
Table 4. Intraoperative outcomes of minimally invasive pancreaticoduodenectomy

\begin{tabular}{|c|c|c|c|c|}
\hline First author (Ref.) & Operative time (min) & Blood loss (mL) & Conversion $n(\%)$ & Transfusion $n(\%)$ \\
\hline Chao Lu (32) & $352.3 \pm 53.2$ & $198.8 \pm 127.6$ & NA & $59(18.4 \%)$ \\
\hline Xueqing Liu (30) & $6.7(2.5-12.0)^{\mathrm{c}} \mathrm{h}$ & $500(100-3000)^{\mathrm{c}}$ & NA & NA \\
\hline Yunqiang Cai (9) & $358(220-495)^{\mathrm{a}}$ & $112(50-800)^{\mathrm{a}}$ & $1(0.4 \%)$ & $11(4.6 \%)$ \\
\hline Hang Zhang (43) & $301 \pm 175$ & $194 \pm 107$ & $3(1.5 \%)$ & $19(9.4 \%)$ \\
\hline Wei Chai (10) & $419.7^{b}$ & $288.1^{\mathrm{b}}$ & NA & NA \\
\hline Qingchun Li (27) & $275.7^{\mathrm{b}}$ & $114.5^{\mathrm{b}}$ & $0(0 \%)$ & $9(6.7 \%)$ \\
\hline Xiaohui Duan (17) & $325.7(220-575)^{\mathrm{c}}$ & $175.9(100-550)^{\mathrm{c}}$ & NA & $2(2.0 \%)$ \\
\hline Guolin Li (25) & $277.5^{\mathrm{b}}$ & $151.7^{\mathrm{b}}$ & NA & NA \\
\hline Tao Zhang (45) & $357.87 \pm 93.28$ & $171.13 \pm 144.46$ & $5(5.0 \%)$ & NA \\
\hline Yong An (7) & $377^{\mathrm{b}}$ & $296^{\mathrm{b}}$ & NA & $18(20.0 \%)$ \\
\hline Yun Liang (28) & $364.6^{\mathrm{b}}$ & NA & $7(8.5 \%)$ & NA \\
\hline Defei Hong (19) & $351.2 \pm 84.1$ & $204.7 \pm 165.9$ & $3(3.8 \%)$ & NA \\
\hline Yusheng Du (16) & $343.5^{\mathrm{b}}$ & $213.2^{\mathrm{b}}$ & $0(0 \%)$ & $5(7.5 \%)$ \\
\hline Shi Chen (14) & $410^{\mathrm{b}}$ & NA & NA & $8(13.3 \%)$ \\
\hline Fangkuan Li (24) & $396.4 \pm 81.9$ & $282.0 \pm 192.4$ & $4(7.4 \%)$ & NA \\
\hline Peng Chen (13) & $487.73 \pm 113.13$ & $360.00 \pm 407.49$ & NA & NA \\
\hline Ronggui Lin (29) & NA & $150.0 \pm 34.6$ & $0(0 \%)$ & NA \\
\hline Jianjun Li (26) & $440.0(382-510)^{\mathrm{c}}$ & NA & $0(0 \%)$ & NA \\
\hline Menghua Dai (15) & $427.16 \pm 78.05$ & $597.06 \pm 327.74$ & $4(11.8 \%)$ & NA \\
\hline Zhigang Wei (37) & $414.5(340-498)^{\mathrm{c}}$ & $420(150-800)^{\mathrm{c}}$ & $0(0 \%)$ & NA \\
\hline Fan Yang (JL) (41) & $4.3 \pm 1.5 \mathrm{~h}$ & $300 \pm 75$ & $0(0 \%)$ & NA \\
\hline Rong Tang (34) & $7.5 \pm 0.8 \mathrm{~h}$ & $326.4 \pm 86.5$ & $0(0 \%)$ & NA \\
\hline Fan Yang (CQ) (42) & $482 \pm 86$ & $400.0(300-800)^{\mathrm{a}}$ & NA & $10(34.5 \%)$ \\
\hline Jianhui Chen (12) & $474.6 \pm 54.2$ & $265.5 \pm 72.6$ & NA & $2(8.0 \%)$ \\
\hline Zuguang Wu (39) & $414.0 \pm 31.0$ & $176.0 \pm 50.4$ & NA & NA \\
\hline Lei Zhang (44) & $317.14 \pm 44.06$ & $523.91 \pm 261.54$ & $0(0 \%)$ & NA \\
\hline Jiacheng Wu (38) & $352 \pm 25$ & $168 \pm 34$ & $0(0 \%)$ & $0(0 \%)$ \\
\hline Zhao Liu (31) & $316(260-410)^{\mathrm{c}}$ & $240(30-1000)^{\mathrm{c}}$ & $1(4.8 \%)$ & NA \\
\hline Qiang Huang (22) & $645.0 \pm 139.9$ & $750.0 \pm 417.6$ & $0(0 \%)$ & NA \\
\hline Qiuya Wei (36) & $407.8 \pm 146.5$ & $309.7 \pm 151.2$ & NA & NA \\
\hline Wentao Gao (18) & $476 \pm 50$ & $439 \pm 228$ & NA & NA \\
\hline Hai Hu (20) & $450 \pm 30$ & $525 \pm 125$ & $1(5.6 \%)$ & $0(0 \%)$ \\
\hline Qinzheng Bai (8) & $470.31 \pm 61.09$ & $568.75 \pm 298.26$ & $0(0 \%)$ & NA \\
\hline Jinmeng $\mathrm{Hu}(21)$ & $459.8 \pm 121.6$ & $178.1 \pm 118.3$ & NA & NA \\
\hline Huanwei Chen (11) & $528.7^{\mathrm{b}}$ & NA & $0(0 \%)$ & $0(0 \%)$ \\
\hline Mingsheng Sun (33) & $280(240-340) c$ & $300(150-1200)^{\mathrm{c}}$ & $2(16.7 \%)$ & NA \\
\hline Jun Xu (40) & $6.5(5-10)^{\mathrm{c}} \mathrm{h}$ & $435(200-800)^{c}$ & $3(25.0 \%)$ & NA \\
\hline Hongbo Wei (35) & $410.2 \pm 85.0$ & $168.2 \pm 87.4$ & $0(0 \%)$ & NA \\
\hline Wu Ji (23) & $7.3 \pm 3.6 \mathrm{~h}$ & $320.0 \pm 123.5$ & $1(10.0 \%)$ & $0(0 \%)$ \\
\hline Total/mean & 370.6 & 278.0 & $35(2.6 \%)$ & $143(10.8 \%)$ \\
\hline
\end{tabular}

${ }^{a}$ Data are expressed as median and interquartile range. ${ }^{b}$ Date are integration from multi-group data. ${ }^{c}$ Data are expressed as mean and range. JL, Jilin Province. CQ, Chongqing City. NA, not applicable.

in choledochojejunostomy were mentioned in 14 and 15 articles respectively. The staple technique occupied the majority in gastroenterostomy $(85.7 \%)$ and running suture was most common in choledochojejunostomy (66.7\%). Besides, 4 authors $(14,17,32,43)$ applied running or interrupted suture selectively depending on diameter of bile duct $(26.7 \%)$. The information of vascular resection and reconstruction was mentioned by 7 authors while 14 studies regarded cases with vascular invasion as contraindications. Overall, 26 cases of vascular involvement were performed with vascular resection and reconstruction.

\subsection{Intraoperative outcomes}

Intraoperative outcomes of MIPD are documented in Table 4, containing operation time, blood loss, conversion rate to laparotomy and intraoperative transfusion rate.

The mean operation time was provided in 37 articles (94.9\%) and ranged from 258 to $645 \mathrm{~min}$, with a WA of $370.6 \mathrm{~min}$. The median operative time was only mentioned in Cai's study (9) with a result of $358 \mathrm{~min}$. The mean blood loss was provided in 33 articles $(84.6 \%)$ ranged from 114.5 to $750 \mathrm{~mL}$, with a WA of $278.0 \mathrm{~mL}$. The median blood loss was mentioned in 2 articles $(9,42)$ with a result of $112 \mathrm{~mL}$ and $400 \mathrm{~mL}$.

The information of conversion rate to laparotomy was available in 25 studies (64.1\%). A total of 35 MIPDs were converted to laparotomy $(2.6 \%)$. The reasons were as follows: uncontrolled vascular bleeding $(n=$ $9,25.7 \%)$, severe adhesions $(n=5,14.3 \%)$, limited working space $(n=1,2.9 \%)$, and unspecified reasons $(n=20,57.1 \%)$. The data of intraoperative transfusion rate was available in 14 articles (35.9\%). A total of 143 MIPDs performed intraoperative transfusion (10.8\%). 
Table 5. Morbidity of minimally invasive pancreaticoduodenectomy

\begin{tabular}{|c|c|c|c|c|c|c|}
\hline First author (Ref.) & Morbidity $n(\%)$ & $\begin{array}{c}\text { Pancreatic } \\
\text { fistula } n(\%)\end{array}$ & $\begin{array}{l}\text { Usage of } \\
\text { ISGPF }\end{array}$ & $\begin{array}{l}\text { Delayed gastric } \\
\text { emptying } n(\%)\end{array}$ & Bile leak $n(\%)$ & $\begin{array}{c}\text { Postoperative } \\
\text { hemorrhage } n(\%)\end{array}$ \\
\hline Chao Lu (32) & $103(32.2 \%)$ & $56(17.5 \%)$ & + & $3(0.9 \%)$ & $12(3.8 \%)$ & $29(9.1 \%)$ \\
\hline Xueqing Liu (30) & $95(31.7 \%)$ & NA & + & $12(4.0 \%)$ & $12(4.0 \%)$ & $28(9.3 \%)$ \\
\hline Yunqiang Cai (9) & NA & $51(21.4 \%)$ & + & $17(7.1 \%)$ & $6(2.5 \%)$ & $3(1.3 \%)$ \\
\hline Hang Zhang (43) & $61(30.2 \%)$ & $29(14.4 \%)$ & + & $23(11.4 \%)$ & $3(1.5 \%)$ & $5(2.5 \%)$ \\
\hline Wei Chai (10) & $67(35.6 \%)$ & $40(21.3 \%)$ & + & $12(6.4 \%)$ & $2(1.1 \%)$ & $3(1.6 \%)$ \\
\hline Qingchun Li (27) & $18(13.4 \%)$ & $16(11.9 \%)$ & + & $2(1.5 \%)$ & $4(3.0 \%)$ & $3(2.2 \%)$ \\
\hline Xiaohui Duan (17) & NA & $23(22.8 \%)$ & + & $4(4.0 \%)$ & $2(2.0 \%)$ & $7(6.9 \%)$ \\
\hline Guolin Li (25) & NA & $63(63.0 \%)$ & + & $5(5.0 \%)$ & $4(4.0 \%)$ & $4(4.0 \%)$ \\
\hline Tao Zhang (45) & $58(58.0 \%)$ & $24(24.0 \%)$ & + & $15(15.0 \%)$ & $11(11.0 \%)$ & $22(22.0 \%)$ \\
\hline Yong An (7) & $19(21.1 \%)$ & NA & + & $5(5.6 \%)$ & $1(1.1 \%)$ & $1(1.1 \%)$ \\
\hline Yun Liang (28) & $22(26.8 \%)$ & NA & + & $1(1.2 \%)$ & NA & $7(8.5 \%)$ \\
\hline Defei Hong (19) & NA & $11(13.8 \%)$ & + & $5(6.3 \%)$ & $6(7.5 \%)$ & $6(7.5 \%)$ \\
\hline Yusheng Du (16) & $20(29.9 \%)$ & $14(20.9 \%)$ & + & $2(3.0 \%)$ & $4(6.0 \%)$ & $1(1.5 \%)$ \\
\hline Shi Chen (14) & $21(35.0 \%)$ & $8(13.3 \%)$ & + & $5(8.3 \%)$ & $5(8.3 \%)$ & $4(6.7 \%)$ \\
\hline Fangkuan Li (24) & $12(24.0 \%)$ & $8(16.0 \%)$ & + & $2(4.0 \%)$ & $2(4.0 \%)$ & $0(0 \%)$ \\
\hline Peng Chen (13) & NA & $10(25.0 \%)$ & - & $5(12.5 \%)$ & NA & $8(20.0 \%)$ \\
\hline Ronggui Lin (29) & NA & $5(14.3 \%)$ & + & $1(2.9 \%)$ & $1(2.9 \%)$ & $1(2.9 \%)$ \\
\hline Jianjun Li (26) & $10(29.4 \%)$ & $2(5.9 \%)$ & + & $0(0 \%)$ & $0(0 \%)$ & $5(14.7 \%)$ \\
\hline Menghua Dai (15) & $15(44.1 \%)$ & NA & + & $0(0 \%)$ & NA & $1(2.9 \%)$ \\
\hline Zhigang Wei (37) & NA & $3(9.1 \%)$ & + & $6(18.2 \%)$ & $1(3.0 \%)$ & NA \\
\hline Fan Yang (JL) (41) & NA & $10(33.3 \%)$ & + & NA & NA & $0(0 \%)$ \\
\hline Rong Tang (34) & $14(48.3 \%)$ & $6(20.7 \%)$ & - & $2(6.9 \%)$ & $3(10.3 \%)$ & $1(3.4 \%)$ \\
\hline Fan Yang (CQ) (42) & $16(55.2 \%)$ & $8(27.6 \%)$ & - & $1(3.4 \%)$ & $1(3.4 \%)$ & $5(17.2 \%)$ \\
\hline Jianhui Chen (12) & $7(28.0 \%)$ & $2(8.0 \%)$ & - & $0(0 \%)$ & $1(4.0 \%)$ & $2(8.0 \%)$ \\
\hline Zuguang Wu (39) & $8(36.4 \%)$ & $4(18.2 \%)$ & - & $0(0 \%)$ & $0(0 \%)$ & $1(4.5 \%)$ \\
\hline Lei Zhang (44) & $4(19.0 \%)$ & $1(4.8 \%)$ & - & $2(9.5 \%)$ & $0(0 \%)$ & $1(4.8 \%)$ \\
\hline Jiacheng Wu (38) & NA & $15(71.4 \%)$ & + & $0(0 \%)$ & $0(0 \%)$ & $0(0 \%)$ \\
\hline Zhao Liu (31) & $5(23.8 \%)$ & $1(4.8 \%)$ & + & $2(9.5 \%)$ & $0(0 \%)$ & $0(0 \%)$ \\
\hline Qiang Huang (22) & $6(30.0 \%)$ & $6(30.0 \%)$ & + & $5(25.0 \%)$ & $1(5.0 \%)$ & $2(10.0 \%)$ \\
\hline Qiuya Wei (36) & NA & $1(5.3 \%)$ & - & $0(0 \%)$ & $1(5.3 \%)$ & $2(10.5 \%)$ \\
\hline Wentao Gao (18) & $10(55.6 \%)$ & $7(38.9 \%)$ & + & $4(22.2 \%)$ & $0(0 \%)$ & $1(5.6 \%)$ \\
\hline Hai Hu (20) & $7(38.9 \%)$ & $4(22.2 \%)$ & + & $0(0 \%)$ & $1(5.6 \%)$ & $3(16.7 \%)$ \\
\hline Qinzheng Bai (8) & NA & $6(37.5 \%)$ & + & NA & NA & NA \\
\hline Jinmeng $\mathrm{Hu}(21)$ & $8(50.0 \%)$ & $5(31.3 \%)$ & + & $2(12.5 \%)$ & $0(0 \%)$ & $0(0 \%)$ \\
\hline Huanwei Chen (11) & NA & $4(26.7 \%)$ & + & $1(6.7 \%)$ & $0(0 \%)$ & $0(0 \%)$ \\
\hline Mingsheng Sun (33) & $3(25.0 \%)$ & $2(16.7 \%)$ & + & $0(0 \%)$ & $1(8.3 \%)$ & $0(0 \%)$ \\
\hline Jun Xu (40) & $1(8.3 \%)$ & $0(0 \%)$ & - & $0(0 \%)$ & $1(8.3 \%)$ & $0(0 \%)$ \\
\hline Hongbo Wei (35) & $4(36.4 \%)$ & NA & - & NA & NA & NA \\
\hline Wu Ji (23) & $1(10.0 \%)$ & $1(10.0 \%)$ & - & $0(0 \%)$ & $0(0 \%)$ & $0(0 \%)$ \\
\hline Total/mean & $615(31.9 \%)$ & $446(20.9 \%)$ & & $144(5.5 \%)$ & $86(3.5 \%)$ & $156(6.0 \%)$ \\
\hline
\end{tabular}

IGSPF, international surgical group definition of pancreatic fistula. JL, Jilin Province. CQ, Chongqing City. NA, not applicable.

\subsection{Short-term outcomes}

Short-term outcomes of MIPD are reported in Table 5 and Table 6, including morbidity, pancreatic fistula (PF) rate, delayed gastric emptying (DGE) rate, bile leak rate, postoperative hemorrhage rate, length of postoperative hospital stay (LOS), Clavien-Dindo $\geq 3$ complication rate, reoperation rate and rate of mortality.

The data of morbidity was included in 27 articles $(69.2 \%)$. The morbidity ranged from 8.3 to $58.0 \%$. Overall, 615 cases of postoperative complications occurred (31.9\%). Particularly, the incidence of pancreatic fistutla was mentioned in 38 articles (97.4\%). The usage of International Surgical Group of Pancreatic Fistula (ISGPF) was employed in 29 articles (76.3\%). In this analysis, we excluded the articles which only presented the data of clinical relevant PF (grade B/C). Incidence of overall PF ranged from 0 to $71.4 \%$. Overall,
446 cases developed PF, giving a total PF rate of $20.9 \%$. The data of DGE was available in 36 articles (92.3\%), and ranged from 0 to $25.0 \%$. In a total of 144 cases DGE occurred (5.5\%). 33 studies $(84.6 \%)$ mentioned bile leak, and ranged from 0 to $11.0 \%$. Overall, 86 cases suffered from bile leak (3.5\%). 36 articles (92.3\%) recorded postoperative hemorrhage rate, and ranged from 0 to $22.0 \%$, which included intraperitoneal and gastrointestinal hemorrhage. In a total of 156 cases postoperative hemorrhage occurred $(6.0 \%)$.

The mean LOS was reported in 30 articles (76.9\%), and ranged from 8.8 to 27.41 days, with a WA of 16.1 days. As for Clavien-Dindo $\geq 3$ complications, only 12 studies listed the results $(30.8 \%)$, which ranged from 0 to $32.5 \%$ and in 116 cases patients developed ClavienDindo $\geq 3$ complications, with an overall rate of $13.4 \%$. The information of reoperation was described in 20 articles $(51.3 \%)$, and ranged from 0 to $17.2 \%$. A total of 
Table 6. Short-term outcomes of minimally invasive pancreaticoduodenectomy

\begin{tabular}{|c|c|c|c|c|}
\hline First author (Ref.) & LOS (days) & Clavien-Dindo $\geq 3$ complications $n(\%)$ & Reoperation $n(\%)$ & Mortality $n(\%)$ \\
\hline Chao Lu (32) & $18.3 \pm 11.7$ & $35(10.9 \%)$ & $17(5.3 \%)$ & $2(0.6 \%)$ \\
\hline Xueqing Liu (30) & $17(6-89)$ & NA & NA & $13(4.3 \%)$ \\
\hline Yunqiang Cai (9) & $10.2(5-19)^{\mathrm{a}}$ & NA & $2(0.8 \%)$ & $1(0.4 \%)$ \\
\hline Hang Zhang (43) & $12.97 \pm 7.21$ & NA & $2(1.0 \%)$ & $1(0.5 \%)$ \\
\hline Wei Chai $(10)$ & $12.3^{\mathrm{b}}$ & NA & $3(1.6 \%)$ & $3(1.6 \%)$ \\
\hline Qingchun Li (27) & $18.9^{\mathrm{b}}$ & NA & NA & $2(1.5 \%)$ \\
\hline Xiaohui Duan (17) & $14.8(8-29)^{\mathrm{c}}$ & $9(8.9 \%)$ & $3(3.0 \%)$ & $1(1.0 \%)$ \\
\hline Guolin Li (25) & $12.9^{\mathrm{b}}$ & NA & NA & NA \\
\hline Tao Zhang (45) & $18 \pm 13.46$ & $22(22.0 \%)$ & $6(6.0 \%)$ & $3(3.0 \%)$ \\
\hline Yong An (7) & $13.3^{\mathrm{b}}$ & NA & $1(1.1 \%)$ & NA \\
\hline Yun Liang (28) & NA & $22(26.8 \%)$ & $5(6.1 \%)$ & $3(3.7 \%)$ \\
\hline Defei Hong (19) & $16.6 \pm 10.1$ & NA & $8(10.0 \%)$ & NA \\
\hline Yusheng Du (16) & $15.4^{\mathrm{b}}$ & $2(3.0 \%)$ & $1(1.5 \%)$ & $0(0 \%)$ \\
\hline Shi Chen (14) & $20.0 \pm 7.4$ & $7(11.7 \%)$ & $2(3.3 \%)$ & $1(1.7 \%)$ \\
\hline Fangkuan Li (24) & $17.17 \pm 6.628$ & NA & NA & NA \\
\hline Peng Chen (13) & $25.86 \pm 12.22$ & $13(32.5 \%)$ & NA & $3(7.5 \%)$ \\
\hline Ronggui Lin (29) & $12.9 \pm 3.2$ & NA & NA & NA \\
\hline Jianjun Li (26) & NA & $3(8.8 \%)$ & $3(8.8 \%)$ & $1(2.9 \%)$ \\
\hline Menghua Dai (15) & NA & NA & $1(2.9 \%)$ & NA \\
\hline Zhigang Wei (37) & NA & NA & NA & NA \\
\hline Fan Yang (JL) (4l) & $16.3 \pm 7.2$ & NA & NA & $0(0 \%)$ \\
\hline Rong Tang (34) & $9.0 \pm 2.1$ & NA & NA & NA \\
\hline Fan Yang (CQ) (42) & $17(15-20)^{\mathrm{a}}$ & NA & $5(17.2 \%)$ & $2(6.9 \%)$ \\
\hline Jianhui Chen (12) & $15.5 \pm 4.2$ & NA & $1(4.0 \%)$ & $0(0 \%)$ \\
\hline Zuguang Wu (39) & $17.3 \pm 2.0$ & NA & NA & NA \\
\hline Lei Zhang (44) & $27.41 \pm 5.82$ & $1(4.8 \%)$ & $1(4.8 \%)$ & NA \\
\hline Jiacheng Wu (38) & $11.3 \pm 2.0$ & NA & NA & $0(0 \%)$ \\
\hline Zhao Liu (31) & NA & NA & NA & NA \\
\hline Qiang Huang (22) & $25.0 \pm 9.3$ & $0(0 \%)$ & $0(0 \%)$ & $0(0 \%)$ \\
\hline Qiuya Wei (36) & $8.8 \pm 2.1$ & NA & NA & $0(0 \%)$ \\
\hline Wentao Gao (18) & $15.5 \pm 6.8$ & NA & NA & $0(0 \%)$ \\
\hline Hai Hu (20) & $16 \pm 4$ & NA & $0(0 \%)$ & $1(5.6 \%)$ \\
\hline Qinzheng Bai (8) & NA & NA & NA & $0(0 \%)$ \\
\hline Jinmeng $\mathrm{Hu}(21)$ & $19.1 \pm 6.0$ & NA & NA & NA \\
\hline Huanwei Chen (11) & $14 \mathrm{~b}$ & NA & NA & $0(0 \%)$ \\
\hline Mingsheng Sun (33) & NA & $2(16.7 \%)$ & $2(16.7 \%)$ & $0(0 \%)$ \\
\hline Jun Xu (40) & 15.0 & NA & NA & $0(0 \%)$ \\
\hline Hongbo Wei (35) & $17.0 \pm 2.2$ & NA & NA & $0(0 \%)$ \\
\hline Wu Ji (23) & $9.6 \pm 4.3$ & $0(0 \%)$ & $0(0 \%)$ & $0(0 \%)$ \\
\hline Total/mean & 16.1 & $116(13.4 \%)$ & $63(3.6 \%)$ & $37(1.7 \%)$ \\
\hline
\end{tabular}

${ }^{\mathrm{a}}$ Data are expressed as median and interquartile range. ${ }^{\mathrm{b}}$ Date are integration from multi-group data. LOS, length of hospital stay. JL, Jilin Province. CQ, Chongqing City. NA, not applicable.

63 cases demanded reoperation (3.6\%). Unfortunately, only 27 studies $(69.2 \%)$ recorded the rate of mortality, which ranged from 0 to $7.5 \%$, and in 37 cases patients died, with an overall postoperative mortality rate of $1.7 \%$.

\subsection{Oncologic outcomes}

The pathology results are shown in Table 7, comprising the rate of malignancy, the number of harvested lymph nodes and the rate of negative tumor margin (R0).

The etiology was described in 35 articles (89.7\%). We regarded ampullary adenocarcinoma, pancreatic ductal adenocarcinoma, distal cholangiocarcinoma, duodenal adenocarcinoma and other malignant tumors clearly identified by the authors as malignancy in our review because of the different attitudes to borderline tumors from diffierent authors. Overall, 2084 cases $(80.7 \%)$ were diagnosed with maligancy. Furthermore, only 7 authors (17.9\%) described the etiology using tumor-node-metastasis (TNM) stage. The mean number of harvested lymph nodes was provided in 20 articles (51.3\%), and ranged from 7.02 to 23.1 , with a WA of 13.5 lymph nodes. Margin status was documented in 25 articles (64.1\%). In 9 articles, the R0 rate was $100 \%$, whereas it ranged from 10 to $99.2 \%$ in the other 16 studies. A total of 79 cases were diagnosed with positive margins out of 1,492 malignancy cases (5.3\%)

\subsection{Comparison of the results of different surgical techniques}

Comparisons of the outcomes between LPD, RPD and open pancreaticoduodenectomy (OPD) are summarized in Table 8. Excluding cumulative data of multiple techniques from three studies, a total of 2,310 LPD, 188 RPD and 779 OPD were accepted for comparison. 
Table 7. Oncologic outcomes of minimally invasive pancreaticoduodenectomy

\begin{tabular}{|c|c|c|c|c|}
\hline First author (Ref.) & Malignancy $^{\mathrm{a}} n(\%)$ & TNM stage & Harvested lymph node & R0 (\%) \\
\hline Chao Lu (32) & $221(69.1 \%)$ & - & NA & NA \\
\hline Xueqing Liu (30) & $258(86.0 \%)$ & - & $12(2-60)$ & $99.3 \%$ \\
\hline Yunqiang Cai (9) & $161(67.6 \%)$ & - & NA & NA \\
\hline Hang Zhang (43) & $147(72.8 \%)$ & + & $9.81 \pm 5.19$ & $99.0 \%$ \\
\hline Wei Chai (10) & $188(100.0 \%)$ & - & $14.9^{\mathrm{b}}$ & $93.6 \%$ \\
\hline Qingchun Li (27) & $134(100.0 \%)$ & + & $23.1^{\mathrm{b}}$ & $92.5 \%$ \\
\hline Xiaohui Duan (17) & $101(100.0 \%)$ & - & $16.7 \pm 4.2$ & $95.0 \%$ \\
\hline Guolin Li (25) & $45(45.0 \%)$ & - & NA & NA \\
\hline Tao Zhang (45) & $78(78.0 \%)$ & - & $7.02 \pm 4.30$ & $100.0 \%$ \\
\hline Yong An (7) & $71(78.9 \%)$ & - & $17.2^{\mathrm{b}}$ & $100.0 \%$ \\
\hline Yun Liang (28) & $60(73.2 \%)$ & - & $12.5^{\mathrm{b}}$ & $95.0 \%$ \\
\hline Defei Hong (19) & $71(88.8 \%)$ & - & NA & $100.0 \%$ \\
\hline Yusheng Du (16) & $60(90.0 \%)$ & - & NA & NA \\
\hline Shi Chen (14) & $38(63.3 \%)$ & + & $13.6 \pm 6.0$ & $97.4 \%$ \\
\hline Fangkuan Li (24) & $46(92.0 \%)$ & - & $11.56 \pm 6.174$ & $97.8 \%$ \\
\hline Peng Chen (13) & $3(75.0 \%)$ & - & NA & $10.0 \%$ \\
\hline Ronggui Lin (29) & $24(68.6 \%)$ & - & $10.6 \pm 4.0$ & $100.0 \%$ \\
\hline Jianjun Li (26) & $34(100.0 \%)$ & - & NA & NA \\
\hline Menghua Dai (15) & $31(91.2 \%)$ & - & NA & $93.5 \%$ \\
\hline Zhigang Wei (37) & $33(100.0 \%)$ & - & NA & $100.0 \%$ \\
\hline Fan Yang $(\mathrm{JL})(41)$ & $26(86.7 \%)$ & - & NA & $92.3 \%$ \\
\hline Rong Tang (34) & $29(100.0 \%)$ & + & NA & NA \\
\hline Fan Yang (CQ) (42) & $20(69.0 \%)$ & + & NA & $90.0 \%$ \\
\hline Jianhui Chen (12) & $25(100.0 \%)$ & - & $12.6 \pm 3.3$ & $100.0 \%$ \\
\hline Zuguang Wu (39) & NA & - & $9.3 \pm 3.0$ & NA \\
\hline Lei Zhang (44) & NA & - & $7.24 \pm 4.81$ & $100.0 \%$ \\
\hline Jiacheng Wu (38) & $21(100.0 \%)$ & - & NA & $100.0 \%$ \\
\hline Zhao Liu (31) & $18(85.7 \%)$ & - & $14(8-26)$ & $94.4 \%$ \\
\hline Qiang Huang (22) & $18(90.0 \%)$ & - & NA & NA \\
\hline Qiuya Wei (36) & $17(89.5 \%)$ & - & $17.7 \pm 6.5$ & $76.5 \%$ \\
\hline Wentao Gao (18) & $13(72.2 \%)$ & - & NA & $69.2 \%$ \\
\hline Hai Hu (20) & $9(50.0 \%)$ & + & $16 \pm 4$ & $88.9 \%$ \\
\hline Qinzheng Bai (8) & $12(75.0 \%)$ & - & NA & NA \\
\hline Jinmeng $\mathrm{Hu}(21)$ & $\mathrm{NA}$ & - & $17.1 \pm 9.7$ & $100.0 \%$ \\
\hline Huanwei Chen (11) & $13(86.7 \%)$ & - & NA & NA \\
\hline Mingsheng Sun (33) & $12(100.0 \%)$ & + & 10 & $100.0 \%$ \\
\hline Jun Xu (40) & $11(91.7 \%)$ & - & NA & NA \\
\hline Hongbo Wei (35) & NA & - & $9.2 \pm 4.0$ & $100.0 \%$ \\
\hline Wu Ji (23) & $9(90.0 \%)$ & - & NA & NA \\
\hline Total/mean & $2,084(80.7 \%)$ & & 13.5 & $1,413(94.7 \%)$ \\
\hline
\end{tabular}

${ }^{a}$ Malignancies include Ampullary adenocarcinoma, Pancreatic ductal adenocarcinoma, Distal cholangiocarcinoma, Duodenal adenocarcinoma and other malignant tumors clearly identified by the authors. ${ }^{b}$ Date are integration from multi-group data. TNM, tumor-node-metastasis. JL, Jilin Province. CQ, Chongqing City. NA, not applicable

The operative time was significantly longer in LPD and RPD groups than in OPD groups. Compared with OPD, LPD shortened LOS significantly. As for morbidity, LPD, RPD and OPD had a result of $30.4 \%$, $46.3 \%$ and $37.9 \%$ respectively. Interestingly, every two of them had a significant difference, and the three techniques had similar results in blood loss, pancreatic fistulas and mortality rates.

\subsection{Comparison of the results of large and small series}

Twenty-one articles documented on 30 or more MIPD $(n$ $=2,318)$, whereas eighteen studies on 29 or fewer MIPD $(n=335)$. (Table 9)

In aspects of operative time and morbidity, the large series had more significant advantages than the small one. No significance, however, was mentioned in blood loss, LOS, PF and mortality rate.

\section{Discussion}

Early in 1994, Gagner described the first case of laparoscopic pylorus-preserving pancreatoduodenectomy for chronic pancreatitis, which marked the beginning of a new era. However, the multiple technical complications covered the benefit of a laparoscopic approach (2). Furthermore, inherent technical limitations of laparoscopy and a long learning curve made this advanced technique develop slowly. Nine years later, Lu reported the first LPD for duodenal papillary cancer in Chinese mainland and achieved comparable outcomes to OPD in perioperative blood loss and short-term recovery (3). Afterwards, an increasing number of reports describing the attempt at MIPD were published. 
Table 8. Comparison of surgical techniques

\begin{tabular}{|c|c|c|c|c|c|c|}
\hline \multirow{2}{*}{ Items } & \multirow{2}{*}{$\operatorname{LPD}(n=2,310)$} & \multirow{2}{*}{$\operatorname{RPD}(n=188)$} & \multirow{2}{*}{ OPD $(n=779)$} & \multicolumn{3}{|c|}{$p$} \\
\hline & & & & LPD vs RPD & LPD vs OPD & RPD vs OPD \\
\hline WA of operative time (min.) & $\begin{array}{c}367.5 \\
(n=2,072)\end{array}$ & $\begin{array}{c}387.6 \\
(n=188)\end{array}$ & $\begin{array}{c}327.7 \\
(n=779)\end{array}$ & NS* & $0.014 *$ & $0.007 *$ \\
\hline WA of blood loss (mL) & $\begin{array}{c}284.7 \\
(n=1912)\end{array}$ & $\begin{array}{c}232.5 \\
(n=128)\end{array}$ & $\begin{array}{c}374.6 \\
(n=613)\end{array}$ & $\mathrm{NS}^{\wedge}$ & $\mathrm{NS}^{\wedge}$ & NS* \\
\hline WA of LOS (days) & $\begin{array}{c}15.7 \\
(n=1811)\end{array}$ & $\begin{array}{c}18.0 \\
(n=188)\end{array}$ & $\begin{array}{c}20.9 \\
(n=733)\end{array}$ & NS* & $0.006^{*}$ & NS* \\
\hline Morbidity (\%) & $\begin{array}{c}528(30.4 \%) \\
(n=1737)\end{array}$ & $\begin{array}{c}87(46.3 \%) \\
(n=188)\end{array}$ & $\begin{array}{c}267(37.9 \%) \\
(n=704)\end{array}$ & $<0.001^{\circ}$ & $<0.001^{\circ}$ & $0.038^{\circ}$ \\
\hline Pancreatic fistula (\%) & $\begin{array}{c}383(21.4 \%) \\
(n=1793)\end{array}$ & $\begin{array}{c}37(19.7 \%) \\
(n=188)\end{array}$ & $\begin{array}{c}150(20.5 \%) \\
(n=731)\end{array}$ & $\mathrm{NS}^{\circ}$ & $\mathrm{NS}^{\circ}$ & $\mathrm{NS}^{\circ}$ \\
\hline Mortality (\%) & $\begin{array}{l}29(1.5 \%) \\
(n=1894)\end{array}$ & $\begin{array}{l}5(2.7 \%) \\
(n=188)\end{array}$ & $\begin{array}{l}14(2.6 \%) \\
(n=537)\end{array}$ & $\mathrm{NS}^{\circ}$ & $\mathrm{NS}^{\circ}$ & $\mathrm{NS}^{\circ}$ \\
\hline
\end{tabular}

* $t$ test unpaired. ${ }^{\wedge}$ Mann-Whitney $U$ test. ${ }^{\circ}$ Chi squared. LPD, laparoscopic pancreaticoduodenectomy. RPD, robotic pancreaticoduodenectomy. OPD, open pancreaticoduodenectomy. WA, weighted average. NS, no significant difference. LOS, length of hospital stay.

Table 9. Comparison of large vs smaller series

\begin{tabular}{lccc}
\hline Items & Large series $(n=2,318)$ & Smaller series $(n=335)$ & $p$ \\
\hline WA of operative time (min.) & $360.0(n=2045)$ & $435.1(n=335)$ & $0.013^{*}$ \\
WA of blood loss (mL) & $266.1(n=1904)$ & $356.0(n=291)$ & $\mathrm{NS}^{\wedge}$ \\
WA of LOS (days) & $16.1(n=1897)$ & $94(35.6 \%)(n=264)$ & $\mathrm{NS}^{*}$ \\
Morbidity (\%) & $466(28.1 \%)(n=1661)$ & $73(22.5 \%)(n=324)$ & $\mathrm{NS}^{\circ}$ \\
Pancreatic fistula (\%) & $373(20.6 \%)(n=1812)$ & $3(1.3 \%)(n=226)$ & $\mathrm{NS}^{\circ}$ \\
Mortality (\%) & $34(1.8 \%)(n=1896)$ & & \\
\hline
\end{tabular}

$* t$ test unpaired. ${ }^{\wedge}$ Mann-Whitney $U$ test. ${ }^{\circ}$ Chi squared. WA, weighted average. NS, no significant difference. LOS, length of hospital stay.

As listed in this systematic review, the maturation of MIPD in Chinese mainland has been extremely quick. The present review is the largest study to evaluate current status of MIPD in both high-volume and low-volume hospitals of Chinese mainland. A total of 2,653 cases were reported in the recent ten years excluding articles of less than 10 cases and initial experience from some highvolume centers. The number of cases reported in the recent two years(from January 2018 to June 2019) was four times than that in the previous years. Although it can't represent the annual cases of MIPD, it demonstrated that MIPD is a research highlight in the Chinese mainland in recent years. In order to highlight distinctive characteristics of MIPD in China mainland and further analyze the difference between Chinese mainland and the world, we present several recent systematic reviews describing the development of MIPD in the world (Table $10)$.

As shown in this table, we have a more favorable outcome in operation time than Nickel (mean:370.6 min vs 417.0min). Probably, this is largely associated with the learning curve and operation mastery. Wang et al were of the view that the minimum number of cases needed to reach technical competency for LPD was 40 cases (48), while Boone et al were first able to reach proficiency after 80 cases in robotic-assisted PD (49). Based on Chinese national conditions, a large number of patients have had a tremendous advantage accumulating surgeon's experience and accelerating surgeon's learning curve, especially in high-volumn centers. While similar results of estimated blood loss were obtained by Nickel's study and this review, Jiang's research offered a worse outcome due to an initial study, which accounted for a large proportion of results.

In terms of morbidity, we found some complications related to MIPD in our review, such as PF, bile leak and postoperative hemorrhage, which were equivalent to those of MIPD as reported in the international reviews. Strangely, more cases suffering DGE were reported in Nickel's study $(21.9 \%$ vs $5.5 \%)$. The reason that contributed to the significant difference maybe was a large number of authors in our review only selectively reported clinically related DGE(grade B/ C) (50). Meanwhile, Nickel only selectively analyzed the clinically related PF because not all trials reported biochemical leaks. Therefore, it's not hard to conclude that we have a slightly better result in overall PF than Nickel, which largely benefited from modified or innovative methods to further reinforce the junction such as purse-string, Bing's anastomosis (9) or Hong's single-stitch method (47). Nonetheless, PF was still the most common postoperative complication no matter in Chinese or international studies. Also, the corrosiveness of pancreatic juice may increase the risk of late postoperative hemorrhage (51). Whatever, more effective and safer anastomosis methods are urgently needed to 
Table 10. Comparison of perioperative and oncological outcomes in previous systematic reviews and this review

\begin{tabular}{|c|c|c|c|c|}
\hline Variables & Nickel (52) & Jiang (54) & Wang (55) & This review \\
\hline \multicolumn{5}{|l|}{ Intraoperative outcomes } \\
\hline Operation time (min) & 417.0 & & & 370.6 \\
\hline Blood loss (mL) & 280.5 & 378.1 & & 278.0 \\
\hline Conversion to laparotomy & & & $61(5.9 \%)$ & $35(2.6 \%)$ \\
\hline Transfusion & & & & $143(10.8 \%)$ \\
\hline \multicolumn{5}{|l|}{ Short-term outcomes } \\
\hline Morbidity & & & $511(49.7 \%)$ & $615(31.9 \%)$ \\
\hline Pancreatic fistula & $20(17.5 \%)$ & $43(15.1 \%)$ & $338(32.9 \%)$ & $446(20.9 \%)$ \\
\hline Delayed gastric emptying & $25(21.9 \%)$ & & $172(16.7 \%)$ & $144(5.5 \%)$ \\
\hline Bile leak & $10(8.8 \%)$ & & $50(4.9 \%)$ & $86(3.5 \%)$ \\
\hline Postoperative hemorrhage & $10(8.8 \%)$ & $24(9.3 \%)$ & $128(12.4 \%)$ & $156(6.0 \%)$ \\
\hline length of hospital stay(days) & 10.6 & 10.0 & 13.5 & 16.1 \\
\hline Clavien-Dindo $\geq 3$ complications & $33(28.9 \%)$ & & $218(21.2 \%)$ & $116(13.4 \%)$ \\
\hline Reoperation & $8(7.0 \%)$ & & $88(8.6 \%)$ & $63(3.6 \%)$ \\
\hline Mortality & $8(7.0 \%)$ & & $25(2.4 \%)$ & $37(1.7 \%)$ \\
\hline \multicolumn{5}{|l|}{ Oncologic outcomes } \\
\hline Harvested lymph node & 14.3 & 19.4 & 10.5 & 13.5 \\
\hline R0 rate & $91(82.0 \%)$ & $695(79.9 \%)$ & $1,004(97.6 \%)$ & $1,413(94.7 \%)$ \\
\hline
\end{tabular}

decrease this formidable complication.

Regarding length of postoperative hospital stay, no significant difference existed between Nickel's and Jiang's studies. Surprisingly, the outcome we pooled was considerably longer than the former two. This difference could, in part, be caused by the different regulations and culture in different countries $(6,52)$. Statistical analysis had been submitted at this point by Boggi with the result of 21.9 days in Europe, 13.0 days in Asia and 9.4 days in North America (O).

The first and the only patient-blinded, randomized clinical trial (LEOPARD-2) was stopped due to a higher mortality rate $(14 \%$ vs $2 \%)$ in the LPD group when compared to the OPD group. However, the four centers included in this trial with a median of $11 \mathrm{LPD}$ (range 6-15) annually. What's more, we couldn't get any information from the original article about how many LPD cases were performed by the surgeons before they participated in the trial and whether they had already finished the learning curve or not.

As for oncologic outcomes, we had a similar result as Nickel in harvested lymph nodes (13.5 vs 14.3), but both were less than Jiang's outcome of 19.4. However, in $\mathrm{R} 0$ resection, we had a significantly better result than the other two. Actually, certainty of evidence in margin status was low. Pathology information has not always been collected according to standardized methodology. Especially for borderline tumors, few authors described their classification criteria.

Wang's study is the Chinese largest multicenter study to date which pooled the data from 1,029 consecutive MIPD patients in 16 high-volume pancreatic centers in China. As shown in the table, we had slight advantages in many factors including conversion to laparotomy, overall and differing morbidity, Clavien-Dindo $\geq 3$ complications, reoperation and mortality. The reasons are not unique. On the one hand, Wang et al pooled the data from January 2010 to August 2016. With more advanced and innovative technologies introduced and the number of surgeons completing the learning curve increasing gradually, it is reasonable to assume that we can acquire better results in either intraoperation or shortterm outcomes. On the other hand, reporting bias in lowvolume hospitals cannot be ignored.

Major venous resection and reconstruction in MIPD has been regarded as a surgical forbidden zone for a long time. In this study, a total of 26 cases of MIPD combined with major venous resection and reconstruction were performed in 7 high-volume pancreatic centers. Besides, in 2018, Cai et al (53) reported an innovative approach to perform the above-mentioned challenging surgical procedures in 18 patients. No 30-day mortality was documented while only one case was converted to laparotomy due to uncontrolled bleeding from the splenic vein. Therefore, it is reasonable to believe that MIPD with major venous resection and reconstruction is technically feasible in selected patients, and with continuous accumulation of surgeons' experience and technological innovations, patients with vascular involvement will no longer be an absolute contraindication for MIPD.

This review is also subject to limitations. First, some technique details including pylorus preservation PD, section of pancreatic neck and long-term oncological outcomes such as overall survival and recurrencefree survival were not described in this study due to a lack of enough original data. Second, the study has not further compared and analyzed the outcomes of MIPD in different periods. Therefore, we can hardly observe the improvement and progress of results from recent years. Third, the quality of evidence is generally limited to cohort studies and case series.

In conclusion, although the developmental stage of MIPD in Chinese mainland was nearly a decade late, 
its development was extremely quick, especially in the recent two years. The operative volume of MIPD in Chinese mainland is in the leading position in the world. Compared with some large international meta-analysis, non-inferior perioperative and short-term oncological outcomes were observed in MIPD of Chinese mainland. What's more, nearly 50 cases were documented in the condition of major vascular resection and reconstruction in Chinese mainland, which represented the operative quality to a certain degree. However, research on survival analysis and phased learning curve outcomes is urgently needed before the innovative surgical techniques are widely accepted.

\section{References}

1. Cameron JL, He J. Two thousand consecutive pancreaticoduodenectomies. J Am Coll Surg. 2015; 220:530-536.

2. Gagner M, Pomp A. Laparoscopic pylorus-preserving pancreatoduodenectomy. Surg Endosc. 1994; 8:408-410.

3. Lu BY, Lu WQ, Cai XY, Wei YX, Jiang HX. Laparoscopic pancreatoduodenectomy for duodenal papillary carcinoma: A case report. Chinese Journal of Minimally Invasive Surgery. 2003; 3:197-198. (in Chinese)

4. Croome KP, Farnell MB, Que FG, Reid-Lombardo KM, Truty MJ, Nagorney DM, Kendrick ML. Total laparoscopic pancreaticoduodenectomy for pancreatic ductal adenocarcinoma: Oncologic advantages over open approaches? Ann Surg. 2014; 260:633-638; discussion 638-640.

5. Gumbs AA, Rodriguez Rivera AM, Milone L, Hoffman JP. Laparoscopic pancreatoduodenectomy: A review of 285 published cases. Ann Surg Oncol. 2011; 18:13351341 .

6. Boggi U, Amorese G, Vistoli F, Caniglia F, De Lio N, Perrone V, Barbarello L, Belluomini M, Signori S, Mosca F. Laparoscopic pancreaticoduodenectomy: A systematic literature review. Surg Endosc. 2015; 29:9-23.

7. An Y, Zhang Y, Liu SY, Cai HH, Chen WB, Wu D, Sun DL, Chen XM. Comparison of the clinical application of three-dimensional and two-dimensional laparoscopic pancreaticoduodenectomy. Zhonghua Wai Ke Za Zhi. 2019; 57:353-357. (in Chinese)

8. Bai QZ, Xu JW, Li F, Liu H, Wang L. The technique of laparoscopic pancreaticoduodenectomy surrounding the portal vein-superior mesenteric vein axis:a report of 16 cases. Journal of Laparoscopic Surgery. 2016; 21:776779. (in Chinese)

9. Cai YQ, Luo H, Li YB, Gao P, Peng B. A novel technique of pancreaticojejunostomy for laparoscopic pancreaticoduodenectomy. Surg Endosc. 2018; 33:15721577.

10. Chai W, Lei B, Meng Y, Zhao XL, Zhang L, Kong DS, Liu RH. Choice of surgical methods and shortterm therapeutic efficacy analysis of laparoscopic pancreaticoduodenectomy. Chinese Journal of Pancreatology. 2019; 19:98-102. (in Chinese)

11. Chen HW, Wang FJ, Deng FW, Li JY, Hu JY. Preliminary experience of laparoscopic pancreaticoduodenectomy.
Chinese Journal of Hepatic Surgery. 2019; 8:212-216.

12. Chen JH, Lin SB, Li F, Shen Y, Zhou EC, Zhang RL. Comparison of short-term efficacy of laparoscopy-assisted and open pancreaticoduodenectomy. Chinese Journal of General Surgery. 2016; 25:401-406. (in Chinese)

13. Chen P, Zhou B, Sun CD, Qiu FB, Zhang BY, Guo $\mathrm{WD}, \mathrm{Xu} Y Z$. Preliminary comparison of perioperative outcomes between minimally invasive and open pancreatoduodenectomy. Journal of Laparoscopic Surgery. 2018; 23:700-704. (in Chinese)

14. Chen S, Chen JZ, Zhan Q, Deng XX, Shen BY, Peng $\mathrm{CH}, \mathrm{Li} \mathrm{HW}$. Robot-assisted laparoscopic versus open pancreaticoduodenectomy: A prospective, matched, midterm follow-up study. Surg Endosc. 2015; 29:3698-3711.

15. Dai MH. Clinical significance of semi-Derotation artery first approach in laparoscopic pancreatoduodenectomy. Chinese Journal of Operative Procedures of General Surgery (Electronic Version). 2018; 12:275-278. (in Chinese)

16. Du YS, Wang J, Li Y, Ma HQ, Liu L, Zhu YX, Zhao WX. Clinical application of a modified pancreatojejunostomy technique for laparoscopic pancreaticoduodenectomy. HPB (Oxford). 2019; 21:1336-1343.

17. Duan XH, Zhou LX, Tian BZ, Yang JH, Li X, Zeng YH, Jiang B, Mao XH. Three-dimensional laparoscopic pancreaticoduodenectomy for periampullary carcinoma: A single surgical team's experience. Chinese Journal of General Surgery. 2017; 26:1127-1132. (in Chinese)

18. Gao WT, Xi CH, Tu M, DaI XL, Guo F, Chen JM, Wei JS, Lu ZP, Wu JL, Jiang KR, Miao Y. Laparoscopic pancreaticoduodenectomy with a novel artery first and uncinate process first approach through Treitz ligament. Zhonghua Wai Ke Za Zhi. 2017; 55:359-363. (in Chinese)

19. Hong DF, Liu YH, Zhang YH, Zhang CW, Wang YC, Sun XD, Zhang W, Wu WD, Wang ZF, Shen GL, Cheng J, Zhang JG, Lu Y. Laparoscopic and hybrid laparoscopic robotic pancreaticoduodenectomy: A report of 80 cases. Chinese Journal of Practical Surgery. 2016; 36:885888,893. (in Chinese)

20. Hu H, Yu X, Hu G, Sun JC, Wang CF, Huang H. Performance experiences in robotic-assisted pancreaticoduodenectomy: A report of 18 cases. Chinese Journal of General Surgery. 2019; 28:260-266. (in Chinese)

21. Hu JM, Dong JX, Qin J, Gao S, Zhao KL, Bai WW, Wang J. Pancreaticoduodenectomy: A single center experience of 118 patients. Chinese Journal of Hepatobiliary Surgery. 2018; 24:542-544. (in Chinese)

22. Huang Q, Yang J, Shao CS, Lin XS, Xie F, Liu $\mathrm{CH}$, Wang H, Lv XW. Totally laparoscopic pancreaticoduodenectomy: Analysis of 20 patients. Journal of Abdominal Surgery. 2017; 30:302-306. (in Chinese)

23. Ji W, Ding K, Kao XM, He CS, Li N, Li JS. Robotic and laparoscopic hybrid pancreaticoduodenectomy: Surgical techniques and early outcomes. Chin Med J (Engl). 2014; 127:3027-3029.

24. Li FK, Wang XM, Sun WD, Hu MH, Wu Q, Hu KT, Ji YH. Comparison of the short-term clinical efficacies of laparoscopic and open pancreaticoduodenectomy. Acta Academiae Medicinae Wannan. 2017; 36:535-538. (in Chinese)

25. Li GL, Lin Q, Zheng SY, Zhou QB, Chen RF. The evaluation of laparoscopic pancreaticoduodenectomy with programmed process. Chinese Journal of Laparoscopic 
Surgery (Electronic Edition). 2018; 11:85-89. (in Chinese)

26. Li JJ, Lu BY, Huang YB. Application of wrapping pancreatic duct-jejunum anastomosis in laparoscopic pancreaticoduodenectomy. Chinese Journal of Digestive Surgery. 2013; 12:116-119. (in Chinese)

27. Li QC, Xue JH, Li WG. Clinical value of arterial first approach in laparoscopic pancreaticoduodenectomy for periampullary tumor. Chinese Journal of Postgraduates of Medicine. 2019; 42:14-18. (in Chinese)

28. Liang Y, Zhao LT, Jiang CY, Hu PF, Wang HW, Cai ZW, Wang W. Laparoscopic pancreaticoduodenectomy in elderly patients. Surg Endosc. 2019. doi: 10.1007/s00464019-06982-w.

29. Lin RG, Huang HG, Chen YC, Lu FC, Lin XC, Yang $\mathrm{HH}$, Fang HZ, Wang CF. Uncinate process resection via anterior "vein-first" combined with right posterior "arteryfirst" approach in laparoscopic pancreaticoduodenectomy: An analysis of 35 cases. Chinese Journal of Practical Surgery. 2018; 38:560-563. (in Chinese)

30. Liu XQ, Xing ZQ, Qin JZ, Duan JY, Feng F, Wang WB, Yan CQ, Liu JH. Results of 300 consecutive laparoscopic pancreaticoduodenectomies: Experience of single institution. Chinese Journal of Practical Surgery. 2018; 38:306-311. (in Chinese)

31. Liu Z, Yu MC, Zhao R, Liu YF, Zeng JP, Wang XQ, Tan JW. Laparoscopic pancreaticoduodenectomy via a reverse-"V" approach with four ports: Initial experience and perioperative outcomes. World J Gastroenterol. 2015; 21:1588-1594.

32. Lu C, Jin WW, Mou YP, Zhou YC, Zhu QC, Shao HL, Chen K, Li SD. Experience on postoperative complications of laparoscopic pancreaticoduodenectomy. Zhonghua Wai Ke Za Zhi. 2018; 56:822-827. (in Chinese)

33. Sun MS. Laparoscopic pancreaticoduodenectomy with uncinate process approach: A report of 12 cases. Chinese Journal of General Surgery. 2015; 24:1227-1231. (in Chinese)

34. Tang R, Zheng H, Chen J, Ding YJ. Analysis of postoperative complications and prognosis of patients with pancreatic head cancer treated by laparoscopic pancreaticoduodenectomy. Chinese Journal of the Frontiers of Medical Science (Electronic Version). 2017; 9:84-87. (in Chinese)

35. Wei HB, Wei B, Zheng ZH, Huang Y, Huang JL, Fang JF. Comparative study of outcomes after laparoscopic versus open pancreaticoduodenectomy. Chinese Journal of Gastrointestinal Surgery. 2014; 17:465-468. (in Chinese)

36. Wei QY, Liu YY, Yan WF, Fan Y, Wang C. A case matched study on laparoscopic versus open pancreaticoduodenectomy. Chinese Journal of Hepatobiliary Surgery. 2016; 22(332-335). (in Chinese)

37. Wei ZG, Wei YX, Huo TY, Liu Y, Yu J, Yan H, Wang GM. Laparoscopic pancreaticoduodenectomy via a "G "-shaped approach: Experience of 33 cases. Chinese Journal of General Surgery. 2018; 33:575-577. (in Chinese)

38. Wu JC, Zhang XW, Yang YS, Wang Y, Fang H, Lin RX. Application value of pancreaticojejunostomy with double-layer continuous suture in total laparoscopic pancreaticoduodenectomy. Chinese Journal of Digestive Surgery. 2018; 17:746-751. (in Chinese)

39. Wu ZG, Deng GM, Zhang YM, Liu HT, Li ZW. An analysis between laparoscopic and open pancreaticoduodenectomy. Lingnan Modern Clinics in Surgery. 2017; 17:678-680. (in Chinese)
40. Xu J, Liu C, Ji YC, Zhai B, Wang D. Report of 12 cases laparoscopic pancreaticoduodenectomy. Journal of Laparoscopic Surgery. 2010; 15:659-661. (in Chinese)

41. Yang F, Liu YH, Liu SY, Zhang W, Jia BX, Wang YC. Clinical application of total laparoscopic pancreatoduodenectomy with inferior-posterior uncinate process and artery first approach. Chinese Journal of Minimally Invasive Surgery. 2018; 18:908-910. (in Chinese)

42. Yang F, Zhong GC, You K, Liu ZJ. Short-term clinical outcomes of laparoscopic pancreaticoduodenectomy versus open pancreatoduodenectomy: A retrospective analysis. Chinese Journal of Bases and Clinics in General Surgery. 2019; 26:685-690. (in Chinese)

43. Zhang H, Guo XJ, Xia J, Zhu F, Shen M, Wang X, Wang M, Qin RY. Comparison of Totally 3-Dimensional Laparoscopic Pancreaticoduodenectomy and Open Pancreaticoduodenectomy. Pancreas. 2018; 47:592-600.

44. Zhang L, Zhang XJ. A Single-center Retrospective Study of Clinical Efficacy of Laparoscopic and Open Pancreaticoduodenectomy. Medical Information. 2019; 32:118-120. (in Chinese)

45. Zhang T, Zhao ZM, Gao YX, Lau WY, Liu R. The learning curve for a surgeon in robot-assisted laparoscopic pancreaticoduodenectomy: A retrospective study in a highvolume pancreatic center. Surg Endosc. 2018; 33:29272933.

46. Barreto SG, Shukla PJ. Different types of pancreaticoenteric anastomosis. Transl Gastroenterol Hepatol. 2017; 2:89.

47. Hong DF, Liu YH, Zhang YH, Wang YC, Wang ZM, Wu WD, Shen GL, Zhang JG, Zhang W, Cheng J, Peng SY. The role of Hong's single-stitch duct to mucosa pancreaticojejunostomy in laparoscopic pancreaticoduodenectomy. Zhonghua Wai Ke Za Zhi. 2017; 55:136-140. (in Chinese)

48. Wang M, Meng L, Cai Y, Li Y, Wang X, Zhang Z, Peng B. Learning curve for laparoscopic pancreaticoduodenectomy: A CUSUM analysis. J Gastrointest Surg. 2016; 20:924-935.

49. Boone BA, Zenati M, Hogg ME, Steve J, Moser AJ, Bartlett DL, Zeh HJ, Zureikat AH. Assessment of quality outcomes for robotic pancreaticoduodenectomy: Identification of the learning curve. JAMA Surg. 2015; 150:416-422.

50. Wente MN, Bassi C, Dervenis C, Fingerhut A, Gouma DJ, Izbicki JR, Neoptolemos JP, Padbury RT, Sarr MG, Traverso LW, Yeo CJ, Buchler MW. Delayed gastric emptying (DGE) after pancreatic surgery: A suggested definition by the International Study Group of Pancreatic Surgery (ISGPS). Surgery. 2007; 142:761-768.

51. Wente MN, Veit JA, Bassi C, Dervenis C, Fingerhut A, Gouma DJ, Izbicki JR, Neoptolemos JP, Padbury RT, Sarr MG, Yeo CJ, Buchler MW. Postpancreatectomy hemorrhage (PPH): An International Study Group of Pancreatic Surgery (ISGPS) definition. Surgery. 2007; 142:20-25.

52. Nickel F, Haney CM, Kowalewski KF, Probst P, Limen EF, Kalkum E, Diener MK, Strobel O, MullerStich BP, Hackert T. Laparoscopic Versus Open Pancreaticoduodenectomy: A Systematic Review and Meta-analysis of Randomized Controlled Trials. Ann Surg. 2019. doi: 10.1097/SLA.0000000000003309.

53. Cai Y, Gao P, Li Y, Wang X, Peng B. Laparoscopic pancreaticoduodenectomy with major venous resection 
and reconstruction: Anterior superior mesenteric artery first approach. Surg Endosc. 2018; 32:4209-4215.

54. Jiang YL, Zhang RC, Zhou YC. Comparison of overall survival and perioperative outcomes of laparoscopic pancreaticoduodenectomy and open pancreaticoduodenectomy for pancreatic ductal adenocarcinoma: A systematic review and meta-analysis. BMC Cancer. 2019; 19:781.
55. Wang M, Peng B, Liu J, et al. Practice Patterns and Perioperative Outcomes of Laparoscopic Pancreaticoduodenectomy in China: A Retrospective Multicenter Analysis of 1029 Patients. Ann Surg. 2019. doi: 10.1097/SLA.0000000000003190.

(Received October 11, 2019; Revised December 12, 2019; Accepted December 20, 2019) 